\title{
Fifty Shades of Gray, Matter: Using Bayesian priors to improve the power of whole-brain voxel- and connexelwise inferences
}

\author{
Krzysztof J. Gorgolewski*, Pierre-Louis Bazin ${ }^{\dagger}$, Haakon Engen ${ }^{\ddagger}$, Daniel S. Margulies* \\ * Max Planck Research Group: Neuroanatomy and Connectivity \\ ${ }^{\dagger}$ Department of Neurophysics \\ ${ }^{\ddagger}$ Department of Social Neuroscience,Max Planck Institute for Human Cognitive and Brain Sciences \\ Leipzig, Germany
}

\begin{abstract}
To increase the power of neuroimaging analyses, it is common practice to reduce the whole-brain search space to a subset of hypothesis-driven regions-of-interest (ROIs). Rather than strictly constrain analyses, we propose to incorporate prior knowledge using probabilistic ROIs (pROIs) using a hierarchical Bayesian framework. Each voxel prior probability of being "of-interest" or "of-non-interest" is used to perform a weighted fit of a mixture model. We demonstrate the utility of this approach through simulations with various pROIs, and the applicability using a prior based on the NeuroSynth database search term "emotion" for thresholding the fMRI results of an emotion processing task. The modular structure of $\mathrm{pROI}$ correction facilitates the inclusion of other innovations in Bayesian mixture modeling, and offers a foundation for balancing between exploratory analyses without neglecting prior knowledge.
\end{abstract}

Keywords-inference; Bayesian inference; fMRI priors; mixture models;

\section{INTRODUCTION}

Many of our neuroimaging studies begin with a regionspecific hypothesis, and yet we conduct whole-brain voxelwise analyses to explore the entire brain for potential signal. While whole-brain analyses tolerably decrease signal-tonoise (SNR), when we move to the space of voxelwise connections, or connexels [1], the quadratic decrease in SNR for full exploration becomes prohibitive (Fig. 1). Effect sizes which were sufficient in voxelwise analyses cannot be used to distinguish signal from noise in the connexel space. Low SNR in connectivity analyses has traditionally been compensated for by using different kinds of regionof-interest (ROI) selection ("seed-based") or data reduction approaches. The search space is thus restricted to only the connections from an a priori ROI drawn on the connectivity matrix. However, this approach completely discards the information outside the ROI, thus excluding even strong signal that does not fall within it. Additionally, analyses using ROI masks are highly sensitive to their size and shape criteria, and do not convey information about the uncertainty of their borders. To balance these interests, we propose a novel approach for performing inference using a two-level hierarchical mixture model. Instead of using a binary ROI

\section{voxels connexels}
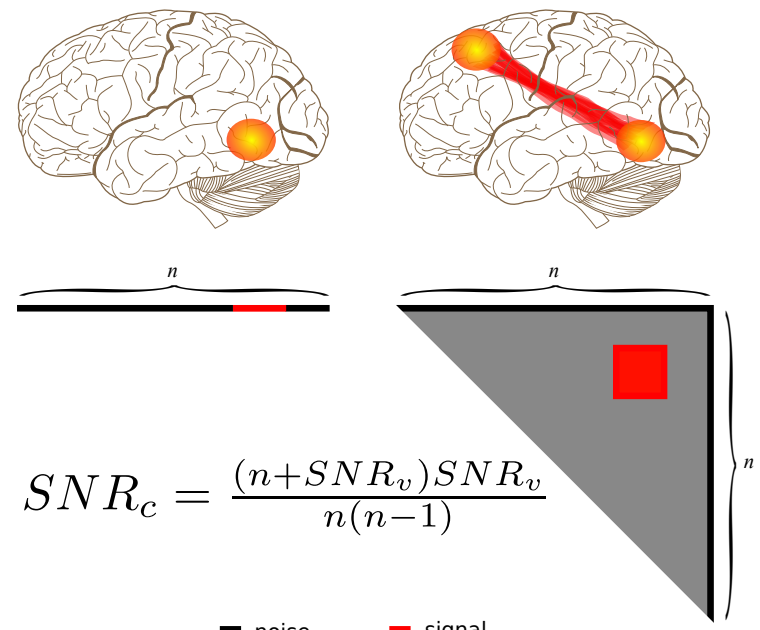

Figure 1. Schematic illustration of whole-brain voxel and connexel analyses. Left: Symbolic representation of flattened voxels (non-active (black) and active (red)). Right: Connectivity matrix where each point corresponds to one connexel. Below: The relation between SNR in the voxelwise $(S N R v)$ and connexelwise $(S N R c)$ cases, where $n$ stands for the number of voxels.

mask we propose a prior probability map ("probabilistic ROI" or $p R O I$ ) with values ranging from 0 to 1 . This map is used to set mixing parameters for probabilistic mixture model distinguishing between voxels-of-interest and those of non-interest (Fig. 2A). In a second level of this hierarchical model, voxels-/connexels-of-interest are modeled as two Gaussian distributions: noise and signal (Fig. 2B). The result of this two-level hierarchical model is inference that incorporates non-binary prior knowledge in the form of pROIs.

\section{Methods}

We propose to formally incorporate prior knowledge into the inference process by using a Bayesian framework. The prior informs the search area, which in turn is subdivided 
into noise and signal. Our hierarchical model consists of two levels. On the first level we model two classes corresponding to voxels-/connexels-of-interest or -of-non-interest.

$$
p(x \mid i)=p\left(m_{1} \mid i\right) p\left(x \mid m_{1}, i\right)+p\left(m_{2} \mid i\right) p\left(x \mid m_{2}, i\right)
$$

Where $p\left(m_{1} \mid i\right)$ are the priors on the search areas (or mixing components of the first level). They are different for each location $(i)$, fixed, and taken set to values based on particular inference assumptions (previous studies, characteristics of different modalities, etc...). Since there are only two components on the first level:

$$
p\left(m_{1} \mid i\right)+p\left(m_{2} \mid i\right)=1
$$

On the second level, the voxels-/connexels-of-interest distribution $\left(p\left(x \mid m_{1}, i\right)\right)$ is described as a mixture of negative gamma (deactivation), Gaussian (noise), and positive gamma (activation) distributions. The location of the two gamma distribution is tied to the estimated mean of the noise component. This level is identical to the model presented in [2].

Parameters of those three distributions (and mixing coefficients) are fitted using a weighted variant of the Expectations-Maximization algorithm [3]: the entire dataset is used but influence of each voxel/connexel on the final mixture is modulated by the $p\left(m_{1} \mid i\right)$ weight in the E-step:

$$
\gamma_{i k}=\frac{p\left(m_{1} \mid i\right) \pi_{k} \mathcal{N}\left(x_{i} \mid \mu_{k}, \sigma_{k}\right)}{\sum_{j=1}^{K} \pi_{j} \mathcal{N}\left(x_{i} \mid \mu_{j}, \sigma_{j}\right)}
$$

Estimates performed in the M-step follow standard form [4]. Additionally the likelihood of data calculated in each step to establish stopping criteria is also weighted:

$$
l=\sum_{i \in S} \log \left(p\left(x_{i} \mid m_{1}, i\right)\right) p\left(m_{1} \mid i\right)
$$

where

$$
S=\left\{i: p\left(m_{1} \mid i\right)>0\right\}
$$

The distribution corresponding to the voxels-/connexelsof-non-interest on the second level is modeled using a simple Gaussian:

$$
p\left(x \mid m_{2}, i\right)=\mathcal{N}(x \mid \mu, \sigma)
$$

Parameters $(\mu, \sigma)$ are being fit using a weight based on $p\left(m_{2} \mid i\right)$ (analogous to Eq. 3).

The actual inference procedure takes two steps (see Fig. 2). First for each voxel/connexel we decide if is ofinterest or non-interest, by comparing the two posterior distributions (without normalization)

$$
p\left(m_{1} \mid i\right)\left(\pi_{N} \mathcal{N}\left(x \mid \mu_{N}, \sigma_{N}\right)+\pi_{S} \mathcal{N}\left(x \mid \mu_{S}, \sigma_{S}\right)\right)
$$

and

$$
p\left(m_{2} \mid i\right) \mathcal{N}(x \mid \mu, \sigma)
$$

For voxels/connexels coming from the of-interest distribution, a similar procedure is used to choose between

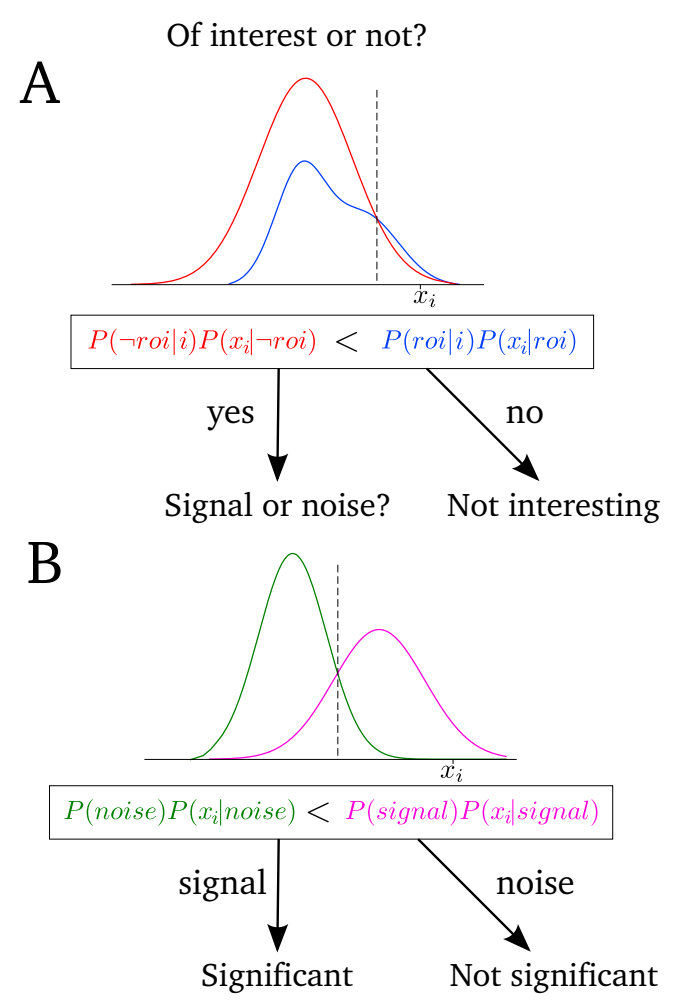

Figure 2. Decision tree within the hierarchical framework. First the fitted distributions are used to classify each voxel/connexel between two categories: "of-interest" or "of-non-interest". In the second step, voxels/connexels-of-interest are classified as "signal" or "noise".

"signal" and "noise" components of the second level mixture. Voxels/connexels with higher posterior probability of coming from "signal" component than "noise" are labeled as "active" (or "significant"). All others (including those rejected on the first level) are labeled as "not active" (or "not significant").

This method is very sensitive to the selection of $p\left(m_{1} \mid i\right)$ and $p\left(m_{2} \mid i\right)$. We define prior map specificity as

$$
\frac{N}{\sum_{i=1}^{N} p\left(m_{1} \mid i\right)}
$$

where $\mathrm{N}$ is the number of connexels/voxels. The higher the specificity of the prior map, the higher the potential SNR, resulting in inference sensitive to smaller effect sizes. On the other side a narrow and (spatially) sharp prior runs the risk of missing the signal if placed in the wrong region. We will investigate these relationships in the following simulations described below.

\section{EXPERIMENTS}

To evaluate the method we have performed a series of simulations using different prior maps. On a two dimensional $100 \times 100$ array two $10 \times 10$ sources of signal were placed (see Fig. 3): one weak (effect size 3) and one strong (effect size 9). Normally distributed $(\sigma=1)$ noise was added 


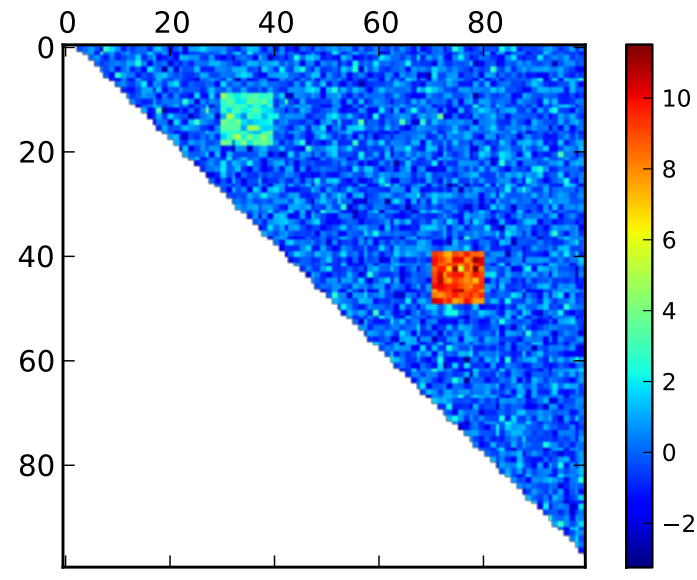

Figure 3. Location and distribution of the simulated signal.

to simulate real conditions. Only upper diagonals of the matrices were used to simulate connectivity analysis.

Six different priors were used: binary prior overlapping with the weak signal (but not the strong signal) equivalent to standard ROI analysis (seed based connectivity), noninformative prior with value 1 at all locations corresponding to full brain analysis and four smooth priors with values ranging from 0.005 (thus always allowing voxels/connexels to be labeled significant if their value is high enough) to 1 (Fig. 4).

The simulation results demonstrate the advantage of using non-binary priors. The "classical ROI" prior fails to find the second signal source, but even the most specific of the non-binary priors (with value 0.005 over the strong signal) does a reasonable job in finding both signals. The advantage diminishes when more weight is put on noisier points, causing the threshold on the second level of inference to rise, and leading to more false negative errors in the region with weak signal (Fig. 4).

It also interesting to look at the weighted SNR (the sum of priors of signal voxels/connexels over sum of all priors values) of each prior map. It is a good estimator of the balance between being specific and correct at the same time. Even though the "classical ROI" priors have a highly weighted SNR, it could be improved if there were prior knowledge of the second source of signal. The inclusion of data points coming from noise decreases the weighted SNR.

We apply the pROI method to thresholding an fMRI dataset acquired during performance of an emotional task. Subjects were exposed to negative and neutral visual imagery with varying uncertainty of the nature of the next stimulus. The prior was generated using the NeuroSynth database (based on the term "emotion" [5]). In comparison to whole-brain analysis, the thresholded map obtained using the pROI delineated activation in the amygdala in voxels that were significant, taking into account their high probability

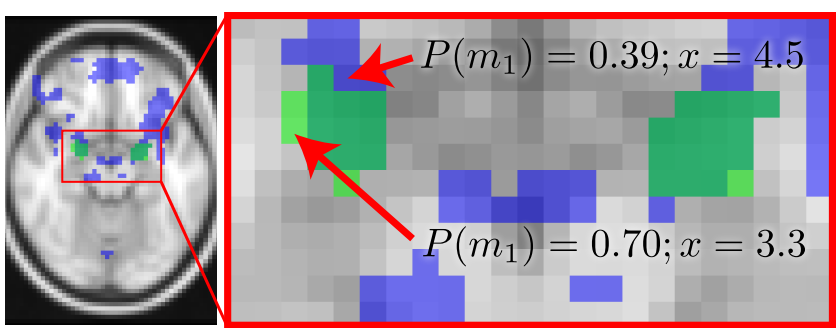

Figure 5. Experiment with emotion task based fMRI. Statistical map was thresholded using full brain non-informative binary prior (transparent blue) and prior based on the "emotion" term in the NeuroSynth database (light green). The NeuroSynth based prior presented cleaner maps. Some voxel were significant only when using one of the priors due to combination of prior probability at given point and value of the statistic (see arrows).

of involvement (Fig. 5).

\section{Discussion}

Restricting the search space to improve SNR has been used in neuroimaging in many forms: brain masks, surfacebased analysis, seed-based connectivity, etc... However, most of these approaches do not incorporate the uncertainty about the location and extent of the restricted areas. The proposed method allows one to vary the degree of "ROIness" of each data-point, encoding the prior knowledge from tissue classification or meta-analyses.

This method, like all statistical tools, could be used as well as abused. Incorporating prior knowledge can increase power, but at the same time if the prior is wrong it can lead to the opposite effect. Additionally, as with binary ROIs, the source of the prior map should be decided a priori, and independent from the data. Using priors estimated from the same dataset as the final inference could lead to false inflation of significance, also known as double dipping [6]. Additionally to avoid circularity, likelihood maps, instead of posterior probability maps, should be included in the future metanalyses when using this method.

Our method provides a modular framework that could be easily extended. For the sake of clarity, we have used a very simple mixture model at the second level, but this model can be augmented using spatial regularization [7] or kernel density estimation of the signal component [8].

Another potential extension involves control over FDR or FNR. At the moment the method presents a classification approach - if there is more evidence that particular voxels/connexel come from the signal distribution than the noise distribution, the voxel/connexel is labeled as significant. This does not control for the potential number of false positive or false negative errors. However, since we are explicitly modeling both signal and noise distributions, we can set the second level threshold to a value corresponding to a desired FDR or FNR level. Since the second level model is fitted using weights, this would be closer to weighted FDR control [9], than the standard [10]. 


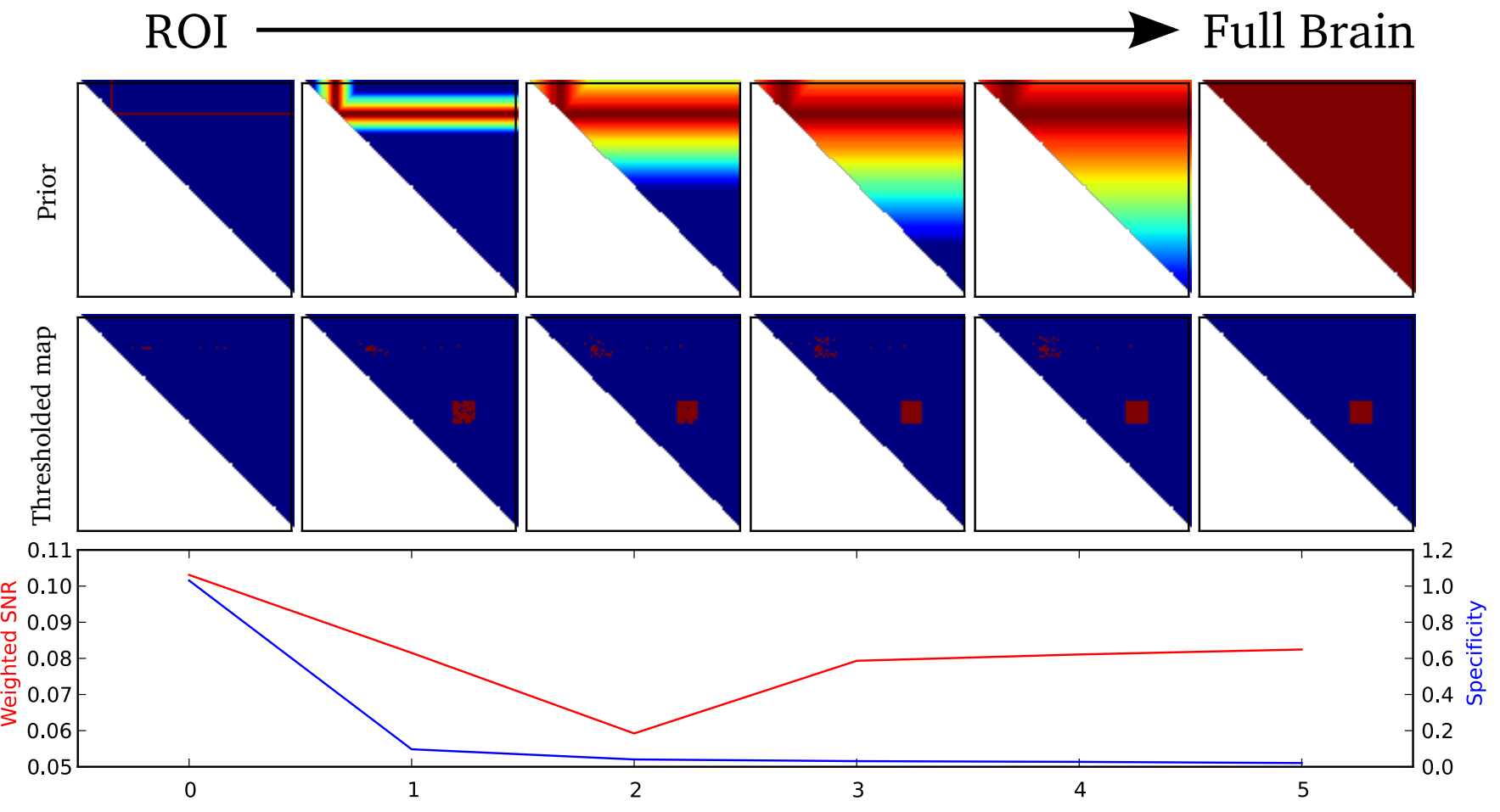

Figure 4. Simulation results. Two sources of were used signal: one weak and one strong (see Fig. 3) and set of prior maps (top row): one representing classical (binary) ROI approach (with the ROI encompassing the weak signal), one representing whole-brain analysis, and four non-binary priors. The "classical ROI" prior misses the strong signal, while the "whole-brain" prior barely finds the weak signal. Only an 'in between' pROI which focuses on the right location, but gives small (but non-zero) chance of finding signal outside of this spot, manages to strike a balance delineating both sources of signal.

\section{CONCLusion}

We propose a new Bayesian inference method that combines the best aspects of ROI and whole-brain analyses increasing sensitivity in areas where signal is hypothesized, while also allowing other signal to be detected. Our model can be easily enhanced by incorporating more sophisticated mixture models and different sources of priors, ranging from those informed by the functional literature to individually derived anatomical features (e.g., tissue segmentation maps, connectivity maps, etc...).

\section{REFERENCES}

[1] K. J. Worsley, J. Cao, T. Paus, M. Petrides, and a. C. Evans, "Applications of random field theory to functional connectivity." Human brain mapping, vol. 6, no. 5-6, pp. 3647 ,

[2] K. Gorgolewski, A. Storkey, M. Bastin, and C. Pernet, "Using a Combination of a Mixture Model and Topological FDR in the Context of Presurgical Planning," in 17th Annual Meeting of the Organization for Human Brain Mapping,

[3] A. Dempster, N. Laird, and D. Rubin, "Maximum likelihood from incomplete data via the EM algorithm," Journal of the Royal Statistical Society: Series B (Statistical Methodology), vol. 39, no. 1, pp. 1-38,

[4] C. Bishop, Pattern recognition and machine learning. Springer New York, 2006,
[5] T. Yarkoni, R. A. Poldrack, T. E. Nichols, D. C. Van Essen, and T. D. Wager, "Large-scale automated synthesis of human functional neuroimaging data," Nature methods, vol. 8, no. 8, pp. 665-70,

[6] N. Kriegeskorte, W. K. Simmons, P. S. F. Bellgowan, and C. I. Baker, "Circular analysis in systems neuroscience: the dangers of double dipping." Nature neuroscience, vol. 12, no. 5 , pp. 535-40,

[7] M. Woolrich, T. Behrens, C. Beckmann, and S. Smith, "Mixture models with adaptive spatial regularization for segmentation with an application to FMRI data," IEEE Transactions on Medical Imaging, vol. 24, no. 1, pp. 1-11,

[8] B. Efron and R. Tibshirani, "Empirical Bayes methods and false discovery rates for microarrays," Genetic epidemiology, vol. 86, no. March, pp. 70-86,

[9] C. Genovese, K. Roeder, and L. Wasserman, "False discovery control with p-value weighting," Biometrika, pp. 509-524,

[10] Y. Benjamini and Y. Hochberg, "Controlling the false discovery rate: a practical and powerful approach to multiple testing," Journal of the Royal Statistical Society. Series B ..., vol. 57 , no. 1 , pp. 289-300, 